\title{
ORIGINAL
}

\section{DETERMINANTES DE LAS CONDUCTAS SEXUALES DE RIESGO EN JOVENES DE CATALUÑA (*)}

\author{
Cinta Folch (1,2), Jose Luis Álvarez (3), Jordi Casabona (1,2), Maria Brotons (4), Xavier Castellsagué \\ $(2,4)$ y Grupo Jóvenes e Internet ${ }^{* *}$.
}

(1) Centre d'Estudis Epidemiològics sobre les Infeccions de Transmissió Sexual i Sida de Catalunya (CEEISCAT), Agència Salut Pública de Catalunya (ASPC), Generalitat de Catalunya, Badalona, España;

(2) CIBER Epidemiología y Salud Pública (CIBERESP), España;

(3) Máster de Salud Pública - Universidad Pompeu Fabra/Universidad Autónoma de Barcelona.

(4) Unidad de Infecciones y Cáncer (UNIC). Programa de Investigación en Epidemiología del Cáncer (PREC). Institut Català d'Oncologia (ICO)-IDIBELL. Barcelona. España.

(*) Agència de Salut Pública de Catalunya, Generalitat de Catalunya; Agència de Gestió d'Ajuts Universitaris i de Recerca - AGAUR (2005/SGR/00505, 2014SGR1077 y 2014SGR2016); Departament d'Universitats, Recerca i Societat de la Informació, de la Generalitat de Catalunya; Departament de Salut Pública, Generalitat de Catalunya; e Instituto de Salud Carlos III (RD12/0036/0056).

${ }^{* *}$ Grupo Jóvenes e Internet: F Xavier Bosch, Silvia de Sanjosé, Laia Bruni, Carmen Cabezas, Luis Urbiztondo, Cristina Agustí y Rossie Lugo (Miembros del Grupo de evaluación del impacto de la vacuna frente al virus del papiloma humano en Cataluña); Laia Ferrer, Alexandra Montoliu, Anna Esteve, Eva Loureiro (CEEISCAT) y Francisco Lupiáñez (Internet Interdiciplinary Institute de la UOC).

Sin conflicto de intereses

\section{RESUMEN}

Fundamentos: La conducta sexual en jóvenes se ve influida por factores externos que pueden favorecer una disminución del control de la situación y mayor desprotección. Objetivo: describir y comparar según sexo la conducta sexual y otros indicadores de salud sexual y reproductiva en jóvenes (16-24 años) en Cataluña e identificar factores asociados a las conductas sexuales de riesgo.

Métodos: Estudio transversal realizado en 2012 mediante encuesta en línea a partir de un panel de población $(\mathrm{n}=800)$. Se identificaron factores asociados al no uso del preservativo (no UP) en la última relación sexual con penetración (RS) mediante modelos de regresión logística multivariantes.

Resultados: E1 76,1\% de los chicos y el 83,3\% de las chicas declararon RS alguna vez $(p=0,012)$. El $39,7 \%$ de los chicos y el $22,5 \%$ de las chicas tuvieron parejas ocasionales, siendo el uso de Internet para contactar parejas del $31,4 \%$ y $10,3 \%$, respectivamente. Entre los factores asociados al no UP durante la última RS destacó no haber usado preservativo en la primera RS $(\mathrm{OR}=2,50$ en chicos y $\mathrm{OR}=3,18$ en chicas). Haber contactado parejas sexuales por Internet se mostró asociado en chicos $(\mathrm{OR}=2,29)$ y haber usado la anticoncepción de emergencia $\geq 3$ veces en chicas $(\mathrm{OR}=3,38)$.

Conclusiones: Se observan diferencias en la conducta sexual de los jóvenes según sexo, siendo el uso del preservativo en la primera relación sexual un buen predictor de su uso en la última. Los chicos presentan mayor número de parejas ocasionales que las chicas y utilizan más las nuevas tecnologías de la información para contactar estas parejas.

Palabras clave: Anticonceptivos. Anticoncepción poscoital. Conducta sexual. Internet. Preservativo. Prevención. Adulto joven.

\section{Correspondencia}

Cinta Folch

Centre d'Estudis Epidemiològics sobre les Infeccions de Transmissió Sexual i Sida de Catalunya (CEEISCAT). Agència de Salut Pública de Catalunya (ASPCAT), Generalitat de Catalunya, Badalona, España. cfolch@iconcologia.net

\section{ABSTRACT}

\section{Factors Associated with Sexual Risk Behaviour Among Young People in Catalonia}

Background: Sexual behavior in young people is influenced by external factors that may increase their vulnerability. Objective: to describe sexual behavior and other indicators of sexual and reproductive health among young people (16 to 24 years) in Catalonia and to identify factors associated with sexual risk behaviors.

Methods: Cross-sectional study in 2012 carried out by an online survey from a panel of population $(n=800)$. Factors associated with not using a condom at last sexual intercourse were evaluated using multivariate logistic regression models.

Results: $76.1 \%$ of boys and $83.3 \%$ of girls reported having had complete sexual intercourse $(\mathrm{p}=0.012)$. The proportion of boys and girls who reported casual partners was $39.7 \%$ and $22.5 \%$, respectively. $31.4 \%$ of boys and $10.3 \%$ of girls reported having contacted partners through Internet. Not using a condom at first sexual intercourse showed significant association with reporting not using a condom in the last sexual intercourse, for both, boys $(\mathrm{OR}=2.50)$ and girls $(\mathrm{OR}=3.18)$. Having contacted partners through the Internet $(\mathrm{OR}=2.29)$ was associated with this risky sexual behavior in boys, and having used the emergency contraception 3 or more times $(\mathrm{OR}=3.38)$ was associated in girls.

Conclusions: There are differences in the sexual behavior of young people by sex, being condom use at first sexual relationship a good predictor of condom use at last sexual intercourse. Boys show a higher number of casual partners than girls, as well as a higher use of the Internet to contact sexual partners.

Keywords: Condoms. Contraception, postcoital. Contraceptive Agents Internet. Prevention. Sexual behavior. Young adult.

DOI: 


\section{INTRODUCCIÓN}

La adolescencia es un período de cambios en el que se inicia la actividad sexual y una etapa en la que la conducta sexual se ve influida por factores externos que pueden favorecer una disminución del control de la situación y una mayor desprotección frente al virus de la inmunodeficiencia humana (VIH), otras infecciones de transmisión sexual (ITS) y embarazos no deseados ${ }^{1}$. Entre los factores que incrementan la vulnerabilidad de los jóvenes destacan el consumo de sustancias como el alcohol y otras drogas, dificultades de negociación del preservativo y el uso de Internet para contactar parejas sexuales, entre otras ${ }^{2-4}$.

En España, al igual que en otros países de Europa, se ha observado en los últimos años un descenso en la edad de inicio de las relaciones sexuales ${ }^{5,6}$. El desapego y/o fracaso escolar, la falta de comunicación con los padres y el consumo de sustancias son algunos de los factores asociados al inicio precoz de las relaciones sexuales (15 años o inferior) en población joven $^{7,8}$.

Los distintos estudios realizados hasta la actualidad han puesto de manifiesto diferencias en las conductas sexuales de los chicos y chicas españoles. En general, los chicos reportan un mayor número de parejas sexuales y un menor uso del preservativo con las parejas ocasionales ${ }^{6,9,10}$. En cambio, las chicas suelen tener relaciones sexuales en el marco de una relación estable ${ }^{6,11}$.

En Cataluña, la prevalencia de Chlamydia trachomatis, en jóvenes usuarios de centros de atención a la salud sexual y reproductiva y centros de atención a jóvenes, muestra un incremento en los últimos años en menores de 25 años del 5,8\% en 2008 al 8,5\% en $2012^{12}$. Por otro lado, entre enero de 2012 y junio de 2013 se reportaron 27 casos de Neisseria gonorrhoea en adolescentes de 13 a 15 años, que, en su mayoría, manifestaron no haber usado el preservativo en su última relación sexual $(80 \%)^{13}$.
El objetivo de este estudio fue describir y comparar la conducta sexual y reproductiva en chicos y chicas de 16 a 24 años en Cataluña e identificar posibles factores asociados a las conductas sexuales de riesgo.

\section{MATERIAL Y MÉTODO}

Estudio transversal realizado en 2012 en jóvenes de 16 a 24 años de edad residentes en Cataluña seleccionados a partir de un panel de población de más de 70.000 personas que cumplía los requisitos que permiten asegurar una muestra representativa. Considerando que la población de 16 a 24 años en Cataluña es de 701.223 individuos, condiciones de máxima indeterminación (prevalencia del $50 \%$ ), un nivel de significación del 5\% y un error de muestreo de 3,54\%, el cálculo del tamaño muestral necesario fue de 800 individuos. La muestra se estratificó por edad, sexo y provincia. La asignación a los estratos se realizó de manera proporcional. Por otro lado, se consideró también el tamaño del municipio de residencia como cuota no cruzada para contrarrestar posibles diferencias entre ámbito rural y urbano (10-14\% de entrevistas en municipios de menos de 5.000 habitantes).

Las personas participantes potenciales se eligieron a partir de bases registradas de diversa temática y recibieron una invitación individual y única para formar parte de la muestra. Se les envió una invitación individual por correo electrónico. Quienes participaron en el estudio también lo hacían, como incentivo, en el sorteo de un iPod. Por si precisaban más información, se les facilitó el contacto de la persona responsable del estudio. El panel incluyó una serie de medidas que garantizaban la calidad del trabajo de campo, como por ejemplo el control del tiempo en responder el cuestionario. Este fue anónimo y de administración en línea. Para su elaboración se tuvo en cuenta la Encuesta Nacional de Salud y Hábitos Sexuales $2003^{6}$ (ENSHS) y se incorporaron los indicadores propuestos por Onusida para evaluar la salud sexual en población joven ${ }^{14}$. Previamente a la implementación del cuestionario se realizó un estudio 
piloto para detectar y solucionar problemas del instrumento de recogida de datos.

El cuestionario solicitaba información sobre características sociodemográficas (edad, sexo, país de origen, nivel de estudios, situación de convivencia y orientación sexual), consumo de alcohol y de drogas ilegales en los últimos 12 meses y conducta sexual (edad de la primera relación sexual con penetración, uso del preservativo en la primera relación sexual, número de parejas sexuales en la vida, uso del preservativo en la última relación sexual de los últimos 12 meses, relaciones sexuales con pareja ocasional en los últimos 12 meses y frecuencia de uso del preservativo, relaciones sexuales homosexuales entre hombres y uso de Internet para contactar parejas sexuales alguna vez). También recogía información sobre otros indicadores de salud sexual y reproductiva (algún diagnóstico de ITS a lo largo la vida, uso de métodos anticonceptivos habituales, uso de la anticoncepción de emergencia en alguna ocasión, número de embarazos e historial de interrupción voluntaria del embarazo), prueba del VIH en alguna ocasión y en los últimos 12 meses. Las preguntas sobre relaciones sexuales hacían referencia a la práctica de la penetración anal y/o vaginal.

La variable dependiente fue "uso del preservativo en la última relación sexual con penetración" (si/no) construida a partir de las respuestas de los jóvenes que declararon haber mantenido relaciones sexuales con penetración (anal y/o vaginal) en los últimos 12 meses.

La comparación de proporciones se realizó mediante la prueba chi $^{2}$ de Pearson y el test exacto de Fisher, y la de medias con el test $t$ de Student. Para identificar los factores asociados al uso del preservativo en la última relación sexual se realizaron modelos de regresión logística univariantes y multivariantes. Las variables con una $p<0,10$ en el modelo univariante fueron analizadas en modelos multivariantes, ajustando el modelo final por la edad. El nivel de significación establecido para todos los análisis fue de 0,05 empleando para su análisis el programa estadístico SPSS versión 17.

El panel cumplía los estándares de calidad internacionales (ESOMAR)100 así como con la Ley Orgánica 15/1999 de 13 de diciembre de Protección de Datosde Carácter Personal ${ }^{15}$. El protocolo del estudio fue aprobado por el Comité de Ética del Hospital Universitario GermansTrias i Pujol.

\section{RESULTADOS}

Del total de participantes (800) el 51,4\% eran hombres y el $48,6 \%$ mujeres, con una edad media de 20 años. El 93,7\% de los chicos y $91,0 \%$ de las chicas eran de origen español $(p=0,155)$ y estudiantes $(67,9 \%$ y $65,6 \%$ de los chicos y chicas respectivamente; $\mathrm{p}=0,180$ ). La convivencia con la familia propia (pareja e hijos) fue del $21,9 \%$ en las chicas y del $10,9 \%$ en los chicos $(\mathrm{p}<0,0001)$, mientras que la convivencia con sus padres y familiares fue mayor en los chicos $(82,0 \%$ frente a $76,1 \% ; p=0,040)$. La mayoría de jóvenes identificó su orientación sexual como heterosexual: $80,8 \%$ y $82,0 \%$ de los chicos y chicas $(\mathrm{p}=0,101)$ (tabla 1$)$.

En los chicos se apreció una frecuencia en el consumo de alcohol en los últimos 12 meses del $72,0 \%$ frente al $66,1 \%$ de las chicas $(p=0,026)$, en cambio no se observaron diferencias estadísticamente significativas con respecto al consumo de drogas ilegales (21,9\% y 20,7\% de los chicos y chicas, respectivamente; $p=0,671)$. La prevalencia del consumo de drogas por vía parenteral alguna vez en la vida fue del $1,0 \%$ en los chicos y del $0,8 \%$ en las chicas (tabla 2 ).

En relación a la conducta sexual, el 76,1\% de los chicos y el 83,3\% de las chicas refirieron haber tenido relaciones sexuales con penetración alguna vez en la vida $(p=0,012)$, sin observarse diferencias estadísticamente significativas en la edad media de inicio según sexo [16,7 años (DE: 1,9) en chicos y 16,5 años $(\mathrm{DE}=1,9)$ en chicas]. El porcentaje de 


\begin{tabular}{|c|c|c|c|c|c|}
\hline \multicolumn{6}{|c|}{$\begin{array}{c}\text { Tabla } 1 \\
\text { Características socio-demográficas de la muestra de jóvenes se- } \\
\text { gún sexo, } 2012\end{array}$} \\
\hline \multirow[t]{2}{*}{ Variables } & \multicolumn{2}{|c|}{ Chicos $n=411$} & \multicolumn{2}{|c|}{ Chicas $\mathrm{n}=389$} & \multirow[t]{2}{*}{$\mathrm{p}$} \\
\hline & $\mathrm{n}$ & $\%$ & $\mathrm{n}$ & $\%$ & \\
\hline Edad media (DE) & 20,2 años & $(2,4)$ & 20,3 años & $(2,5)$ & 0,455 \\
\hline Grupo de edad & & & & & 0,989 \\
\hline 16 a 19 años & 153 & 37,2 & 145 & 37,3 & \\
\hline 20 a 24 años & 258 & 62,8 & 244 & 62,7 & \\
\hline País de origen & & & & & 0,155 \\
\hline Autóctono & 385 & 93,7 & 354 & 91,0 & \\
\hline Inmigrante & 26 & 6,3 & 35 & 9,0 & \\
\hline Ocupación o situación profesional* & & & & & 0,180 \\
\hline Trabajo activo & 62 & 15,1 & 77 & 19,8 & \\
\hline Estudiante & 279 & 67,9 & 255 & 65,6 & \\
\hline En paro, otro** & 70 & 17,0 & 57 & 14,7 & \\
\hline Nivel de estudios máximo & & & & & 0,002 \\
\hline Estudios primarios & 15 & 3,6 & 13 & 3,3 & \\
\hline ESO FP Bachillerato & 346 & 84,2 & 292 & 75,1 & \\
\hline Universitarios & 50 & 12,2 & 84 & 21,6 & \\
\hline Ha vivido sólo* & & & & & 0,232 \\
\hline No & 401 & 97,6 & 384 & 98,7 & \\
\hline $\mathrm{Si}$ & 10 & 2,4 & 5 & 1,3 & \\
\hline \multicolumn{6}{|l|}{ Convivencia con otras personas ${ }^{* * *}$} \\
\hline Con familia propia (pareja/hijos) & 45 & 10,9 & 85 & 21,9 & \\
\hline Con padres y/o familiares & 337 & 82,0 & 296 & 76,1 & 0,040 \\
\hline Con amigos y/o otros & 78 & 19,0 & 59 & 15,2 & 0,153 \\
\hline Orientación sexual & & & & & 0,101 \\
\hline Homosexual & 36 & 8,8 & 26 & 6,7 & \\
\hline Bisexual & 22 & 5,4 & 29 & 7,5 & \\
\hline Heterosexual & 332 & 80,8 & 319 & 82,0 & \\
\hline Otra/cuestionando/Prefiere no contestar & 21 & 5,0 & 15 & 3,8 & \\
\hline
\end{tabular}

jóvenes que refirió haber usado el preservativo en la primera relación sexual fue similar en ambos sexos: $85,2 \%$ y $86,1 \%$ en chicos y chicas, respectivamente $(\mathrm{p}=0,102)$.

Entre los jóvenes que habían mantenido relaciones sexuales con penetración alguna vez, un mayor porcentaje de chicas que de chicos declaró haber tenido relaciones con una pareja heterosexual en los últimos 12 meses: $90,3 \%$ frente a 77,6\% $(\mathrm{p}<0,001)$. Entre estos, el $75,6 \%$ de los chicos y el $58,4 \%$ de las chicas refirieron haber usado el preservativo en su última relación sexual heterosexual $(p<0,001)$. Los chicos presentaron mayor frecuencia de relaciones sexua- les con parejas ocasionales que las chicas $(39,7 \%$ frente a $22,5 \% ; \mathrm{p}<0,001)$, sin diferencias estadísticamente significativas en el uso consistente del preservativo con estas parejas: $68,8 \%$ en chicos y $70,3 \%$ en chicas $(p=0,842)$. El porcentaje de chicos que refirió haber tenido relaciones sexuales con otros hombres en los últimos 12 meses fue del 10,5\%, de los cuales el 54,5\% afirmó haber usado el preservativo en su última relación sexual homosexual (tabla 3 ).

El 31,4\% de los chicos y el 10,3\% de las chicas que habían tenido relaciones sexuales alguna vez refirieron haber usado Internet para conseguir parejas ( $\mathrm{p}<0,001)$, siendo su- 


\begin{tabular}{|c|c|c|c|c|c|}
\hline \multicolumn{6}{|c|}{$\begin{array}{c}\text { Tabla } 2 \\
\text { Consumo de drogas ilegales y alcohol en la muestra de jóvenes } \\
\text { según sexo, } 2012\end{array}$} \\
\hline \multirow{2}{*}{ Variables } & \multicolumn{2}{|c|}{$\begin{array}{c}\text { Chicos } \\
(\mathrm{n}=411)\end{array}$} & \multicolumn{2}{|c|}{$\begin{array}{c}\text { Chicas } \\
(\mathrm{n}=389)\end{array}$} & \multirow{2}{*}{$\mathrm{p}$} \\
\hline & $\mathrm{n}$ & $\%$ & $\mathrm{n}$ & $\%$ & \\
\hline $\begin{array}{l}\text { Frecuencia del consumo de alcohol (últimos } 12 \\
\text { meses) }\end{array}$ & & & & & 0,026 \\
\hline Más de una vez al mes & 293 & 72,0 & 257 & 66,1 & \\
\hline Menos de una vez al mes & 58 & 14,3 & 84 & 21,6 & \\
\hline Nunca & 56 & 13,8 & 48 & 12,3 & \\
\hline Consumo de drogas ilegales (últimos 12 meses) & & & & & 0,671 \\
\hline $\mathrm{Si}$ & 89 & 21,9 & 79 & 20,7 & \\
\hline No & 317 & 78,1 & 303 & 79,3 & \\
\hline \multicolumn{6}{|l|}{ Tipo de droga consumida (últimos 12 meses)* } \\
\hline Hachís, marihuana o cannabis & 86 & 96,6 & 73 & 92,4 & 0,309 \\
\hline Cocaína & 10 & 11,2 & 13 & 16,5 & 0,326 \\
\hline GHB o éxtasis líquido & 3 & 3,4 & 1 & 1,3 & 0,623 \\
\hline Drogas de diseño [pastis, pirules] & 7 & 7,9 & 4 & 5,1 & 0,464 \\
\hline SPEED o anfetaminas & 10 & 11,2 & 11 & 13,9 & 0,599 \\
\hline Alucinógenos (LSD, ácido, Tripi) & 8 & 9,0 & 4 & 5,1 & 0,324 \\
\hline Inhalables volátiles & 3 & 3,4 & 0 & 0,0 & 0,248 \\
\hline Consumo de drogas inyectadas (alguna vez) & & & & & 1,000 \\
\hline $\mathrm{Si}$ & 4 & 1,0 & 3 & 0,8 & \\
\hline No & 404 & 99,0 & 386 & 99,2 & \\
\hline
\end{tabular}

perior el porcentaje de chicos que manifestó haber tenido relaciones sexuales con alguien conocido a través de Internet (el 14,6\% y $5,4 \%$ de los chicos y chicas refirieron haber tenido relaciones con más de dos parejas, respectivamente; $p<0,001)$. No se observaron diferencias estadísticamente significativas en cuanto al uso del preservativo con la última pareja sexual contactada a través de Internet $(81,9 \%$ en chicos y $76,7 \%$ en chicas; $p=0,387$ ) (tabla 3). Entre los chicos, los que refirieron haber tenido alguna vez relaciones sexuales con hombres reportaron con mayor frecuencia haber usado Internet alguna vez para conseguir parejas $(62,8 \%$ frente a $27,7 \%$, respectivamente; $p<0,001$ ).

El $17,6 \%$ de los chicos y el $13,7 \%$ de las chicas se habían realizado alguna vez la prueba para el VIH/sida $(p=0,134)$, siendo positivo el resultado en $4(5,6 \%)$ chicos. No se observaron diferencias estadísticamente significativas en la prevalencia autodeclarada de ITS según sexo $(2,5 \%$ de los chicos y $2,6 \%$ de las chicas refirieron haber sido diagnosticados en alguna ocasión; $p=0,909$ ).

El 10,7\% de las chicas que declararon haber tenido relaciones sexuales había estado alguna vez embarazada, con diferencias estadísticamente significativas según la edad $(4,3 \%$ en chicas de 16 a 19 años y $13,3 \%$ en chicas de 20 años o más; $p=0,018$ ) (tabla 4).

La mitad de las chicas refirió haber usado la anticoncepción de emergencia en alguna ocasión $(27,9 \%$ una vez, $13,2 \%$ dos veces y $9,7 \%$ tres veces o más). Por otro lado, la frecuencia de interrupciones voluntarias del embarazo fue superior en chicas de 20 a 24 años con respecto a las más jóvenes $(9,3 \%$ frente a $3,2 \% ; p=0,062$ ) (tabla 4$)$. 


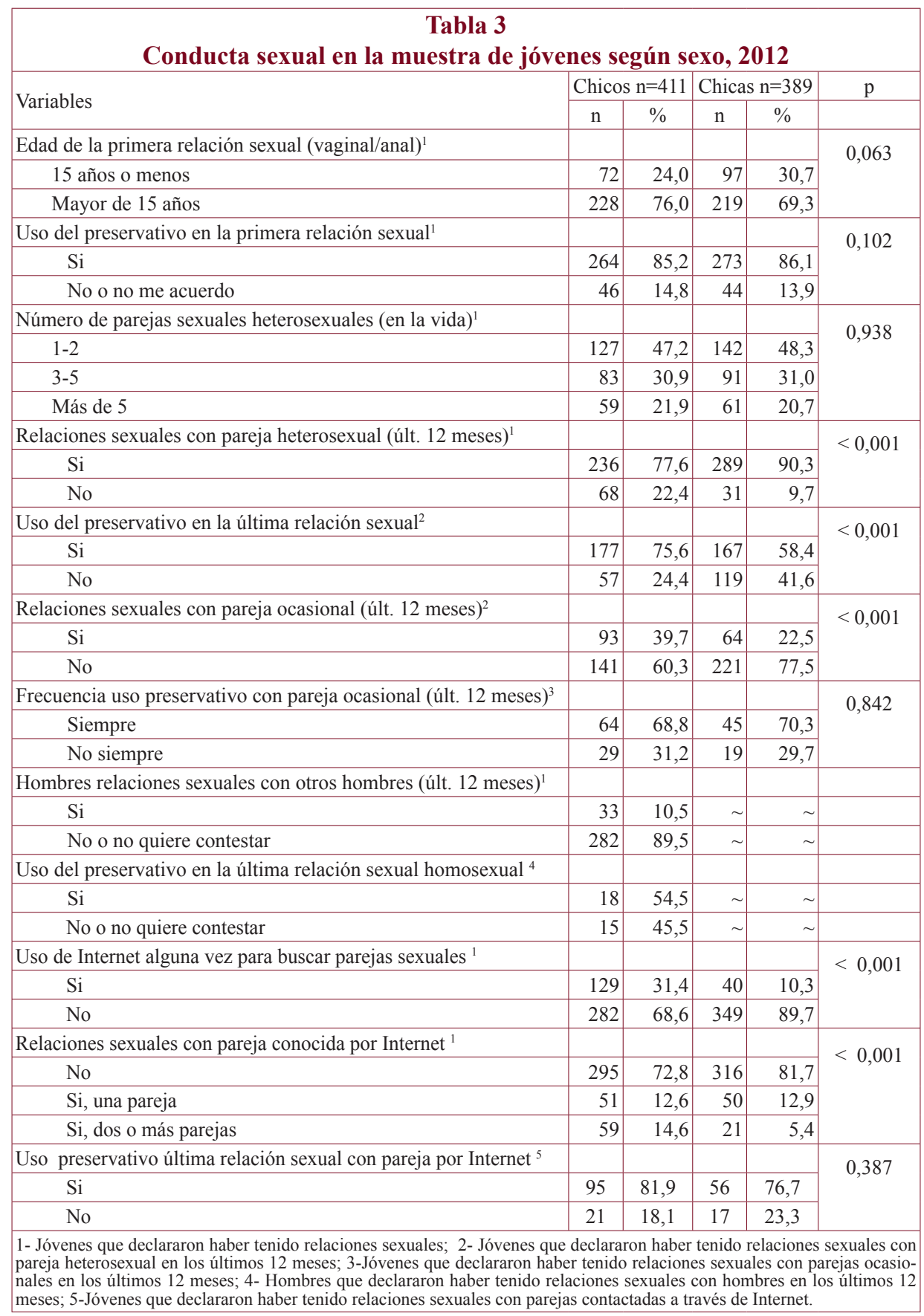




\begin{tabular}{|c|c|c|c|c|c|c|c|}
\hline \multicolumn{8}{|c|}{$\begin{array}{c}\text { Tabla } 4 \\
\begin{array}{c}\text { Indicadores de salud sexual y reproductiva en chicas } \\
\text { según edad, } 2012^{*}\end{array}\end{array}$} \\
\hline & \multicolumn{2}{|c|}{ Total } & \multicolumn{2}{|c|}{$16-19$ años } & \multicolumn{2}{|c|}{ 20-24 años } & \multirow{2}{*}{$\mathrm{p}$} \\
\hline & $\mathrm{n}$ & $\%$ & $\mathrm{n}$ & $\%$ & $\mathrm{n}$ & $\%$ & \\
\hline Has estado embarazada alguna vez ${ }^{*}$ & & & & & & & 0,018 \\
\hline $\mathrm{Si}$ & 34 & 10,7 & 4 & 4,3 & 30 & 13,3 & \\
\hline No & 284 & 89,3 & 89 & 95,7 & 195 & 86,7 & \\
\hline Uso de la anticoncepción de emergencia* & & & & & & & 0,486 \\
\hline Nunca & 157 & 49,2 & 52 & 55,3 & 105 & 46,7 & \\
\hline Una & 89 & 27,9 & 25 & 26,6 & 64 & 28,4 & \\
\hline Dos & 42 & 13,2 & 10 & 10,6 & 32 & 14,2 & \\
\hline Tres o más & 31 & 9,7 & 7 & 7,4 & 24 & 10,7 & \\
\hline \multicolumn{8}{|l|}{ Alguna interrupción voluntaria del embarazo* } \\
\hline $\mathrm{Si}$ & 24 & 7,5 & 3 & 3,2 & 21 & 9,3 & 0,062 \\
\hline No & 295 & 92,5 & 90 & 96,8 & 205 & 90,7 & \\
\hline
\end{tabular}

\begin{tabular}{|c|c|c|c|c|c|c|c|}
\hline \multicolumn{8}{|c|}{$\begin{array}{c}\text { Tabla } 5 \\
\text { Factores asociados a no haber usado el preservativo en la última relación sexual } \\
\text { según sexo (últimos } 12 \text { meses). Análisis de regresión logística multivariante }\end{array}$} \\
\hline & \multirow{2}{*}{ Edad } & \multicolumn{3}{|c|}{ Chicos } & \multicolumn{3}{|c|}{ Chicas } \\
\hline & & OR & IC $95 \%$ & $\mathrm{p}$ & OR & IC $95 \%$ & $\mathrm{p}$ \\
\hline & 16 a 19 & 1 & & & 1 & & \\
\hline & 20 a 24 & 2,29 & $1,05-5,00$ & 0,038 & 1,94 & $1,10-3,40$ & 0,021 \\
\hline \multirow{2}{*}{ Convivencia familia propia* } & Sin ellos & 1 & & & $\sim$ & $\sim$ & $\sim$ \\
\hline & Pareja, hijos & 2,20 & $1,04-4,64$ & 0,039 & $\sim$ & $\sim$ & $\sim$ \\
\hline \multirow{2}{*}{ Uso del preservativo en la primera relación sexual } & $\mathrm{Si}$ & 1 & & & 1 & & \\
\hline & No usó o no recuerda & 2,50 & $1,21-5,16$ & 0,013 & 3,18 & $1,48-6,87$ & 0,003 \\
\hline \multirow{2}{*}{ Prueba del VIH (últimos 12 meses) } & No & $\sim$ & $\sim$ & $\sim$ & 1 & & \\
\hline & $\mathrm{Si}$ & $\sim$ & $\sim$ & $\sim$ & 3,53 & $1,11-11,22$ & 0,032 \\
\hline \multirow{2}{*}{ Relaciones sexuales con pareja conseguida por internet ${ }^{*}$} & No & 1 & & & $\sim$ & $\sim$ & $\sim$ \\
\hline & $\mathrm{Si}$ & 2,29 & $1,25-4,18$ & 0,007 & $\sim$ & $\sim$ & $\sim$ \\
\hline \multirow{2}{*}{ Uso de la anticoncepción de emergencia (alguna vez) } & 0,1 о 2 & $\sim$ & $\sim$ & $\sim$ & 1 & & \\
\hline & Tres o más & $\sim$ & $\sim$ & $\sim$ & 3,38 & $1,43-7,98$ & 0,006 \\
\hline Últimos 12 meses. & & & & & & & \\
\hline
\end{tabular}

En el modelo final de regresión logística multivariante (tabla 5), los factores asociados a no haber utilizado el preservativo en la última relación sexual para ambos chicos y chicas fueron: tener más de 20 años $(\mathrm{OR}=$ 2,29 y 1,94, respectivamente) y no haber usado el preservativo en la primera relación sexual $(\mathrm{OR}=2,50$ y 3,18 respectivamente). En el caso de los chicos, convivir con la familia propia (pareja e hijos) $(\mathrm{OR}=2,20) \mathrm{y}$ haber contactado parejas sexuales por Inter- net $(\mathrm{OR}=2,29)$ mostraron también una asociación significativa con esta conducta sexual de riesgo. Por otro lado, las chicas que se habían realizado la prueba del VIH en los últimos 12 meses $(\mathrm{OR}=3,53)$ y las que habían usado la anticoncepción de emergencia tres o más veces en el último año $(\mathrm{OR}=3,38)$ tenían significativamente más probabilidad de no haber usado el preservativo en la última relación sexual. 


\section{DISCUSIÓN}

Los resultados de este estudio confirman que un elevado porcentaje de los jóvenes de entre 16 y 24 años en Cataluña refieren haber tenido relaciones sexuales con penetración alguna vez. Entre estos, aproximadamente una cuarta parte inició sus relaciones sexuales antes de los 16 años, resultado consistente con otros estudios recientes realizados en España en población joven ${ }^{6,16}$. En cuanto al uso del preservativo entre los jóvenes en la primera relación sexual es superior al observado en otros países europeos ${ }^{5}$.

Al igual que en otros trabajos realizados en nuestro país ${ }^{9-11}$, se observan diferencias en la conducta sexual de los jóvenes según sexo. Las chicas presentan mayor actividad sexual en los últimos 12 meses y un menor uso del preservativo en la última relación sexual que los chicos. Estas diferencias pueden ser debidas en parte al hecho que los chicos tengan mayor número de parejas ocasionales y por tanto se protejan más que las chicas. Otros trabajos ya han mostrado como las chicas tienen relaciones con pareja estable en mayor proporción que los chicos y con estas parejas se protegen menos debido a que en las relaciones sexuales con su pareja estable conceden una gran importancia al vínculo afectivo, buscando profundizar la relación de pareja bajo un contexto amoroso con una percepción del riesgo de infección por VIH/ ITS disminuida ${ }^{6,10}$.

Un hallazgo de nuestro estudio es que el porcentaje de chicos que afirmó haber tenido relaciones sexuales con otros chicos es más elevado que el observado en otros estudios nacionales ${ }^{6,16}$, en parte debido a la mayor concentración de comunidad gay en grandes ciudades como Barcelona y Madrid donde se puede vivir la homosexualidad con mayor anonimato ${ }^{17}$.

Este estudio pone de manifiesto por primera vez en España el uso que hacen los jóvenes de Internet para contactar parejas sexuales, práctica descrita previamente en
Estados Unidos ${ }^{18,19}$. El empleo de Internet desde temprana edad logra que los jóvenes sean expertos en el uso de sus aplicaciones, es la vía para realizar visitas a sitios de contenido sexual, ampliar su conjunto de conocidos, tener cibersexo y conseguir parejas sexuales. En este sentido, los resultados de nuestro estudio confirman que los chicos utilizan más este medio para contactar parejas que las chicas, en particular los chicos que afirman haber tenido relaciones con otros hombres alguna vez.

Entre los factores asociados tanto en chicos como en chicas al no uso del preservativo en la última relación sexual se encuentra la edad y el uso del preservativo en la primera. En cuanto a la edad, son diversos los estudios que apuntan un descenso del uso del preservativo al incrementarse la edad, en parte debido al uso de otros métodos anticoncepti$\operatorname{vos}^{6,11}$. Por otro lado, el uso del preservativo en la primera relación ha mostrado previamente ser un buen predictor del uso del preservativo del acto sexual más reciente ${ }^{20}$. En este sentido, un estudio prospectivo realizado en Estados Unidos concluyó que no solo los adolescentes que habían usado el preservativo en su primera relación sexual tenían más probabilidad de haberlo usado durante su vida sexual más reciente sino que también presentaban menor prevalencia de ITS con respecto los adolescentes que refirieron no haber usado este método en su primer contacto sexual ${ }^{21}$.

Los chicos que declararon convivir con la familia propia usaron el preservativo en la última relación sexual en menor proporción que el resto. Esto podría explicarse al haber cambiado su estado de convivencia proceden a diversificar los métodos anticonceptivos, disminuyendo el uso del preservativo argumentando enamoramiento, confianza plena y la libertad de utilizar otras opciones ${ }^{22}$.

Por último, haber contactado parejas sexuales por Internet se mostró asociado en los chicos a un menor uso del preservativo en la última relación sexual. Existen nume- 
rosos estudios que muestran una posible asociación entre el uso de Internet para contactar parejas sexuales y la mayor prevalencia de prácticas sexuales de riesgo, especialmente en el colectivo de hombres que practican sexo con hombres ${ }^{23-25}$. Un reciente estudio realizado en adolescentes norteamericanos observó una asociación entre haber usado Internet para contactar parejas y ciertas conductas que implicaban un mayor riesgo de infección, como tener un mayor número de parejas sexuales ${ }^{19}$.

En las chicas, haberse realizado la prueba del VIH en el último año se asoció a no haber usado el preservativo en la última relación sexual. Esta posible asociación podría estar relacionada con la mayor percepción de riesgo que presentan las chicas jóvenes después de haber presentado una exposición de riesgo, tal y como muestra la ENSHS de $2003^{6}$.

Finalmente, las chicas que declararon haber usado la anticoncepción postcoital 3 o más veces en la vida mostraron una asociación con no haber usado el preservativo en la última relación sexual respecto las chicas que sí lo habían usado, con una frecuencia menor o que no lo habían usado nunca. No obstante, no podemos saber si el hecho de no usar preservativo lleva al uso de la anticoncepción de emergencia o, bien, saber que se puede recurrir a este método conlleva acabar no usando el preservativo. En cualquier caso, el porcentaje de chicas que utiliza la anticoncepción de emergencia y, por tanto, que han mantenido una relación sexual no protegida es superior al observado en otros estudios españoles ${ }^{10,26}$, siendo un indicador indirecto del potencial alto grado de exposición a patógenos de transmisión sexual en esta población. Aunque otros trabajos muestran que la disponibilidad de este método anticonceptivo no incrementa las conductas de riesgo en los jóvenes $^{27}$, en España se observa un posible incremento en el porcentaje de mujeres que solicita este método en más de una ocasión ${ }^{28}$.

Entre las limitaciones del estudio destaca en primer lugar el posible sesgo de selección de los participantes en relación a los jóvenes sin acceso a Internet. Sin embargo, consideramos que este sesgo fue minoritario ya que datos del Instituto Nacional de Estadística muestran que más del $90 \%$ de los adolescentes de 15 años usan actualmente ordenadores con conexión a Internet. El hecho de tratarse de un estudio anónimo de administración en línea, lo que garantiza la privacidad, seguramente ayuda a disminuir la infradeclaración de ciertas conductas de riesgo socialmente poco aceptadas. Por otro lado, al ser un estudio transversal no se pueden establecer relaciones de causalidad entre los factores estudiados y la variable dependiente.

En definitiva, los resultados obtenidos en este estudio nos indican aspectos diferenciales en los determinantes de las conductas sexuales de riesgo de los chicos y chicas catalanes. Es imprescindible integrar la prevención de los embarazos no deseados en un marco más amplio de la educación afectivo-sexual. Además estas estrategias deberían implementarse a temprana edad, incrementando así la probabilidad de adquirir hábitos saludables y el uso sistemático del preservativo. En este sentido, las nuevas Tecnologías de la Información (TIC) se perfilan como una herramienta más a tener en cuenta para la prevención y promoción de la salud en jóvenes.

\section{BIBLIOGRAFÍA}

1. Equipo de trabajo interinstitucional (ETI) sobre VIH y los jóvenes. Breves guías globales. Intervenciones relacionadas con el VIH para jóvenes. Nueva York: UNFPA; 2008.

2. Slater C, Robinson A. Sexual health in adolescents. Clin Dermatol. 2014; 32:189-95.

3. Vivancos R, Abubakar I, Hunter PR. Sex, drugs and sexually transmitted infections in British university students. Int J STD AIDS. 2008; 19:370-7.

4. Labay Matías M, Labay Guerrero A, Labay Guerrero M. Internet, Sexo y Adolescentes: Una nueva realidad. Encuesta a jóvenes universitarios españoles. Rev Ped Aten Prim. 2011; 13:225-32.

5. Currie C, Zanotti C, Morgan A, Currie D, de Looze M, Roberts C, et al. Social determinants of health and wellbeing among young people. Health Behaviour in Schoolaged Children (HBSC) study: international report from the 2009/2010 survey. Copenhagen: WHO Regional Office for Europe; 2012 (Health Policy for Children and Adolescents, No. 6). 
6. Instituto Nacional de Estadística y Ministerio de Sanidad y Consumo. Encuesta de Salud y Hábitos sexuales. Madrid: Instituto Nacional de Estadística; 2004. Disponible en: http://www.ine.es/inebmenu/mnu_salud.htm

7. Madkour AS, Farhat T, Halpern CT, Godeau E, Gabhainn SN. Early adolescent sexual initiation as a problem behavior: A comparative study of five nations. J Adolesc Health. 2010; 47:389-98.

8. Lavikainen HM, Lintonen T, Kosunen E. Sexual behavior and drinking style among teenagers: a population-based study in Finland. Health Promot Int. 2009; 24:108-19.

9. Rodriguez Carrión J, Traverso Blanco C. Conductas sexuales en adolescentes de 12 a 17 años de Andalucía. Gac Sanit. 2012; 26:519-24.

10. Puente D, Zabaleta E, Rodríguez-Blanco T, Cabanas M, Monteagudo M, Pueyo MJ, et al. Gender differences in sexual risk behaviour among adolescents in Catalonia, Spain. Gac Sanit. 2011; 25:13-9.

11. Faílde Garrido J, Lameiras-Fernández, M BimbelaPedrola J. Prácticas sexuales de chicos y chicas españoles de 14-24 años de edad. Gac Sanit. 2008; 22:511-9.

12. SIVES 2012: Sistema Integrat de Vigilància Epidemiològica de la SIDA/VIH/ITS a Catalunya. Barcelona: Generalitat de Catalunya. Agència de Salut Pública de Catalunya, 2013. (Documento técnico; 21). Disponible en: http://www.ceeiscat.cat/documents/SIVES_2012. pdf

13. Vives N, Lugo R, López E, García de Olalla P, Minguell S, Barrabeig I, et al. Increase in gonorrhoea among very young adolescents, Catalonia, Spain, January 2012 to June 2013. Euro Surveill. 2013;18(33):pii=20560.

14. Joint United Nations Programme on HIV/AIDS (UNAIDS). Global AIDS Response progress reporting: monitoring the 2011 political declaration on HIV/AIDS: guidelines on construction of core indicators: 2012 reporting. Ciudad donde se edita:Vasa; 2011 p. 1-233.

15. Boletín Oficial del Estado. Ley Orgánica 15/1999 de 13 de diciembre de Protección de Datos de Carácter PersonaL BOE núm. 298 de 12/12/1999.

16. García-Vega E, Menéndez Robledo E, Fernández García P, Cuesta Izquierdo, M. Sexualidad, Anticoncepción y Conducta Sexual de Riesgo en Adolescentes. Int J Psychol Res. 2012; 5:79-87.

17. Encuesta "on-line" europea para hombres que tienen relaciones sexuales con hombres: (EMIS): resultados en España [CD-ROM]. Madrid: Ministerio de Sanidad, Servicios Sociales e Igualdad;2013.
18. Zaldívar S, Díez I. Nuevas Dimensiones, Nuevas adicciones: La Adiccion al al Sexo en Internet. Interv Psicosoc. 2009; 18:255-68.

19. Buhi ER, Klinkenberger N, McFarlane M, Kachur R, Daley EM, Baldwin J, et al. Evaluating the Internet as a sexually transmitted disease risk environment for teens: findings from the communication, health, and teens study. Sex Transm Dis. 2013; 40:528-33.

20. Stulhofer A, Bacak V, Ajdukovi D, Graham C. Understanding the association between condom use at first and most recent sexual intercourse: An assessment of normative, calculative, and habitual explanations. Soc Sci Med. 2010; 70:2080-4

21. Shafii T, Stovel K, Holmes K. Association between condom use at sexual debut and subsequent sexual trajectories: a longitudinal study using biomarkers. Am J Public Health. 2007; 97:1090-5.

22. Bimbela JL, Jimenez JM, Alfaro N, Gutiérrez P, March JC. Uso del profiláctico entre la juventud en sus relaciones de coito vaginal. Gac Sanit. 2002; 16:298-307.

23. Liau A, Millett G, Marks G. Meta-analytic examination of online sex-seeking and sexual risk behavior among men who have sex with men. Sex Transm Dis. 2006; 33:576-84.

24. Fernández-Dávila P, Zaragoza Lorca K. Trust and Sexual Interaction: The Significance of the Internet on the Sex life and Sexual Risk Behaviors Of Gay and Bisexual Men in Spain. Int J Sex Health. 2011; 23: 139-55.

25. Folch C, Muñoz R, Zaragoza K, Casabona J. Sexual risk behaviour and its determinants among men who have sex with men in Catalonia, Spain. Euro Surveill 2009; 14(47):pii=19415. Disponible en: www.eurosurveillance.org/ViewArticle.aspx?Articleld=19415

26. López-Amorós M, Schiaffino A, Moncada A, Pérez G. Factores asociados al uso autodeclarado de la anticoncepción de emergencia en la población escolarizada de 14 a 18 años de edad. Gac Sanit. 2010; 24:404-9.

27. Sander PM, Raymond EG, Weaver MA. Emergency contraceptive use as a marker of future risky sex, pregnancy, and sexually transmitted infection. Am J Obstet Gynecol. 2009; 201:146.e1-6.

28. Santamaría Rodríguez T, Crespo del Pozo AV, Cid Prados A, Gontán García-Salamanca MJ, González Pérez M, Baz Collado C, et al. Anticoncepción de emergencia: perfil de las usuarias y características de la demanda. SEMERGEN. 2006;32:321-4. 


\section{Anexo 1 \\ Cuestionario utilizado en la investigación \\ Jóvenes e Internet}

\section{Sección I: Características socio-demográficas:}

1. Sexo

Hombre

Mujer

2. ¿Cuál es tu fecha de nacimiento?

3. ¿En qué país naciste?

$\square$ España $\square$ Municipio de residencia:

Otro país $\square$ Especificar país:

4. ¿Cuál es el máximo nivel de estudios que has completado?

$\square$ Sin estudios

Estudios primarios

Enseñanza secundaria obligatoria (ESO)

Enseñanza de grado medio de Formación Profesional

Bachillerato

Enseñanza de grado superior de Formación Profesional

Estudios universitarios

5. En relación al trabajo realizado durante los últimos 12 meses, ¿en qué situación has estado la mayor parte del tiempo?

$\neg$ Trabajando

Parado pero buscado trabajo anteriormente

Parado buscando mi primer empleo

Estudiando

$\neg$ Otra situación (especificar):

6. ¿Con cuáles de las siguientes personas has vivido la mayor parte del tiempo en los últimos 12 meses? Marcar todas las que apliquen

$\square$ Solo

Cónyuge/pareja

Padres o tutor

Hijos propios

Hijos de la pareja o cónyuge

Otros familiares adultos

Amigos/compañeros de piso

$\neg$ Otras personas

Sección II: Consumo de alcohol y drogas

7. ¿Con qué frecuencia has consumido bebidas alcohólicas durante los últimos 12 meses?

$\square$ No he consumido

Diariamente

De cuatro a seis días a la semana

De dos a tres días a la semana

Un día a la semana

Un día al mes

Menos de un día al mes 
8. ¿Has consumido drogas ilegales (hachís, cocaína, éxtasis, etc.) alguna vez durante los últimos 12 meses?

$\neg$ Sí

$\square$ No $\square$ pasa a la pregunta 12

Prefiero no responder

9. ¿Qué tipo de drogas has consumido en alguna ocasión durante los últimos 12 meses? Marcar todas las que apliquen Hachís o marihuana [Cannabis, hierba, chocolate, porro, costo]

Cocaína en polvo [Cocaína, perico, farlopa, clorhidrato de cocaína]

GHB o éxtasis líquido

Éxtasis o drogas de diseño [pastis, pirulas]

SPEED o anfetaminas [metanfetamina, anfetas, ice, cristal]

Alucinógenos [LSD, ácido, tripi, setas mágicas, ketamina, special-K]

Inhalables volátiles [Cola, pegamento, disolvente, poppers, nitritos]

Otras drogas. Especificar:

Prefiero no responder

10.Algunas personas consumen drogas mediante inyección. ¿Alguna vez en la vida te has inyectado alguna droga?

$\neg$ Sí

No

Prefiero no responder

\section{Sección III: Conducta sexual}

11.¿Has tenido alguna vez relaciones sexuales completas (vaginales o anales)?

$\square$ Sí

No $\square$ pasa a la pregunta 27

Prefiero no responder

12.¿A qué edad tuviste tu primera relación sexual (vaginal o anal)?

$\square$ Edad en años:

Prefiero no responder

13.¿Usaste el preservativo en esa primera relación sexual completa?

$\square$ Sí

No

No lo recuerdo

Prefiero no responder

14.¿Cómo definirías tu orientación sexual?

$\neg$ Homosexual

Bisexual

Heterosexual

Otra

Me lo estoy cuestionando / no lo sé

Prefiero no responder

\section{Sección III-A: Relaciones heterosexuales}

15.A lo largo de la vida, ¿aproximadamente con cuantas parejas distintas has tenido relaciones sexuales (vaginales o anales)?

$\square_{-} \ldots$ parejas

Prefiero no responder 
16.En los últimos 12 meses, ¿has tenido relaciones sexuales (vaginales o anales) con una pareja heterosexual?

$\square$ Sí

$\square$ No $\square$ pasa a la pregunta 0

$\square$ Prefiero no responder

17.En los últimos 12 meses, ¿aproximadamente con cuantas parejas diferentes has tenido relaciones sexuales (vaginales o anales)?

$\square$ _ parejas

$\square$ Prefiero no responder

18.¿Utilizaste el preservativo en tu última relación sexual (vaginal o anal)?

$\square$ Sí

$\square$ No

$\square$ Prefiero no responder

19.En los últimos 12 meses, ¿has tenido relaciones sexuales (vaginales o anales) con una pareja que no fuera tu pareja estable?

$\square$ Sí

$\square$ No $\square$ pasa a la pregunta 0

$\square$ Prefiero no responder

20.¿Con qué frecuencia utilizaste el preservativo con estas parejas ocasionales en las relaciones sexuales (vaginales o anales) de los últimos 12 meses?

Siempre

Ocasionalmente

Nunca

Prefiero no responder

\section{Sección III-B: Relaciones homosexuales}

21.A lo largo de la vida, ¿aproximadamente con cuantos hombres diferentes has tenido relaciones sexuales con penetración?

_-_ hombres

Prefiero no responder

22.En los últimos 12 meses, ¿has tenido relaciones sexuales con penetración con otros hombres?

Sí

No $\square$ pasa a la pregunta 25

Prefiero no responder

23.En los últimos 12 meses, ¿aproximadamente con cuántos hombres diferentes has tenido relaciones sexuales con penetración?

_- hombres

Prefiero no responder

24.¿Utilizaste el preservativo en tu última relación sexual con penetración con una pareja masculina?

$\square$ Sí

No

$\square$ Prefiero no responder 


\section{Sección IV: Salud sexual y reproductiva}

25.¿Te han diagnosticado en alguna ocasión alguna infección de transmisión sexual (ITS)?

$\square$ Sí

$\square$ No $\square$ pasa a la pregunta 27

$\square$ Prefiero no responder

26.Si es que sí, ¿Cuál/es? Marcar todas las que apliquen

Infección por clamidia

Gonorrea

Sífilis

Tricomonas

Herpes genital

Verrugas genitales o condilomas

Candidiasis o hongos

Vaginitis bacteriana

Hepatitis B

10.Uretritis no específica

11.Otra ITS, especificar:

27. ¿A dónde acudirías en caso de tener una verruga genital u otra infección de transmisión sexual?

$\square$ Médico de cabecera

Dermatólogo

Centro de atención a la Salud sexual y reproductiva

Hospital

Otro lugar:

28.En tu última relación sexual, ¿tú o tu pareja utilizasteis algún método anticonceptivo? Marcar todas las que apliquen

$\square$ Anticonceptivos orales, píldora

DIU o dispositivo intrauterino

Diafragma

Preservativo

Ligadura de trompas

Retirada o marcha atrás

Método Ogino

Otro, especificar:

No hemos utilizado ningún método anticonceptivo

Sección IV-I: Sección exclusiva para mujeres

29.¿Has estado embarazada en alguna ocasión?

$\square$ Sí

No $\square$ pasa a la pregunta 32

Prefiero no responder

30.¿Has tenido alguna vez alguna interrupción voluntaria del embarazo?

$\square$ Sí

$\square$ No

Prefiero no responder 
31.¿Ha utilizado alguna vez la píldora del día después para evitar un embarazo?

$\square$ Sí $\square$ Número de veces:

$\square$ No

$\square$ Prefiero no responder

32.¿Te has realizado alguna vez la prueba del VIH o del sida?

$\square$ Sí $\square$ pasa a la pregunta 34

1 No

$\square$ Prefiero no responder

33. ¿Cuál ha sido el motivo principal por el que no te has realizado la prueba del VIH?

$\square$ No quiero saberlo

No sé donde ir a hacerme la prueba

Siempre tengo relaciones sexuales protegidas

Me preocupa el impacto que pueda tener en mi vida sexual o personal

Otros motivos:

$\square$ Prefiero no responder

34.¿Cuánto hace que te realizaste la última prueba del VIH o del sida?

$\square$ Hace menos de 12 meses

$\square$ Entre uno y dos años

Entre dos y cinco años

Hace más de cinco años

Prefiero no responder

35.¿Cuál fue el resultado de tu última prueba del VIH o del sida?

$\square$ Negativo

$\square$ Positivo

No fui a recoger los resultados

No lo quiero decir

MUCHAS GRACIAS POR TU COLABORACION 